\title{
Risk of Cancer after Lower Urinary Tract Infection: A Population-Based Cohort Study
}

\author{
Chia-Hung Huang $1,2,+$, Ying-Hsiang Chou ${ }^{1,3,4, \dagger}$, Han-Wei Yeh ${ }^{5}$, Jing-Yang Huang ${ }^{6} \oplus$, \\ Shun-Fa Yang $1,6, *(\mathbb{D}$ and Chao-Bin Yeh $7,8, *(\mathbb{D})$ \\ 1 Institute of Medicine, Chung Shan Medical University, Taichung 402, Taiwan; \\ hchiahung@gmail.com (C.-H.H.); hideka.chou@gmail.com (Y.-H.C.) \\ 2 Division of Nephrology, Department of Internal Medicine, Lin Shin Hospital, Taichung 402, Taiwan \\ 3 Department of Medical Imaging and Radiological Sciences, Chung Shan Medical University, \\ Taichung 402, Taiwan \\ 4 Department of Radiation Oncology, Chung Shan Medical University Hospital, Taichung 402, Taiwan \\ 5 School of Medicine, Chang Gung University, Taoyuan City 333, Taiwan; george66889@gmail.com \\ 6 Department of Medical Research, Chung Shan Medical University Hospital, Taichung 402, Taiwan; \\ wchinyang@gmail.com \\ 7 Department of Emergency Medicine, School of Medicine, Chung Shan Medical University, \\ Taichung 402, Taiwan \\ 8 Department of Emergency Medicine, Chung Shan Medical University Hospital, Taichung 402, Taiwan \\ * Correspondence: ysf@csmu.edu.tw (S.-F.Y.); sky5ff@gmail.com (C.-B.Y.) \\ + These authors contributed equally to the work.
}

Received: 3 January 2019; Accepted: 28 January 2019; Published: 30 January 2019

\begin{abstract}
To investigate the association among lower urinary tract infection (UTI), the type and timing of antibiotic usage, and the subsequent risk of developing cancers, especially genitourinary cancers (GUC), in Taiwan. This retrospective population-based cohort study was conducted using 2009-2013 data from the Longitudinal Health Insurance Database. This study enrolled patients who were diagnosed with a UTI between 2010 and 2012. A 1:2 propensity score-matched control population without UTI served as the control group. Multivariate analysis with a multiple Cox regression model was applied to analyze the data. A total of 38,084 patients with UTI were included in the study group, and 76,168 participants without UTI were included in the control group. The result showed a higher hazard ratio of any cancer in both sexes with UTI (for males, adjusted hazard ratio $(\mathrm{aHR})=1.32 ; 95 \%$ confidence interval $(\mathrm{CI})=1.12-1.54$; for females, $\mathrm{aHR}=1.21 ; 95 \% \mathrm{CI}=1.08-1.35)$. Patients with UTI had a higher probability of developing new GUC than those without UTI. Moreover, the genital organs, kidney, and urinary bladder of men were significantly more affected than those of women with prior UTI. Furthermore, antibiotic treatment for more than 7 days associated the incidence of bladder cancer in men ( $7-13$ days, $\mathrm{aHR}=1.23,95 \% \mathrm{CI}=0.50-3.02 ;>14$ days, $\mathrm{aHR}=2.73$, $\mathrm{CI}=1.32-5.64)$. In conclusion, UTI is significantly related to GUC and may serve as an early sign of GUC, especially in the male genital organs, prostate, kidney, and urinary bladder. During UTI treatment, physicians should cautiously prescribe antibiotics to patients.
\end{abstract}

Keywords: urinary tract infection; genitourinary organs cancer; retrospective cohort study

\section{Introduction}

Urinary tract infection (UTI) is one of the most common infectious diseases and the main cause of community-acquired and nosocomial infections at admission [1]. Patients with UTI usually present with acute clinical UTI or chronic uncomplicated or complicated UTI. Complicated UTI involve individuals with a condition or more resistant pathogen that increases the risk of failing treatment 
with functional, metabolic, or structural abnormalities [2]. Moreover, the UTI incidence in women is much higher than that in men, but the majority are simple or uncomplicated UTI that occur in healthy or nonpregnant women [3]. In women, the urethra is close to the vagina and rectum, which can lead to the unintentional introduction of fecal flora into the urinary tract, the most common cause of frequent occurrence of UTI. Furthermore, the risk of bacteria colonization increases in postmenopausal women because of the loss of vaginal $\mathrm{pH}$ physiological tuning ability [4].

Lower UTI includes cystitis and prostatitis, and upper UTI include pyelonephritis. The Infectious Diseases Society of America observed a UTI prevalence of $1 \%-5 \%$ in healthy and premenopausal women and $1.9 \%-9.5 \%$ in pregnant women. Symptoms of lower urinary tract are common in men, and the prevalence increases with age. Up to $90 \%$ of men aged 50-80 years may suffer from troublesome lower urinary tract symptoms [5].

Inflammation is a main factor in cancer development, but studies on the relationship between UTI and cancer risks are scant [6]. A recent study demonstrated a relationship between UTI and genitourinary cancers (GUC) and confirmed that recurrent UTI are a risk factor for urinary bladder cancer [7]. Moreover, the relationship between UTI and other tumors is important because UTI-caused inflammatory response is a systemic symptom. In addition, UTI can be diagnosed on the basis of a combination of symptoms and a positive urine analysis or culture [8]. Thus, multiple antimicrobial therapies, including treatment with cephalosporin, quinolone, ampicillin, beta-lactam, amoxicillin, nitrofurantoin, sulfamethoxazole, and trimethoprim, can be prescribed $[9,10]$. Thus, using antibiotics is necessary for UTI treatment. Although evidence for the relationship between UTI and cancer risks is rare, it is reasonable to hypothesize that UTI disorders may still lead to cancers, especially GUC. Therefore, using a nationwide database, this study investigated the association between UTI and antibiotic usage influencing the risk of cancer.

\section{Materials and Methods}

\subsection{Data Source}

A national large-scale database, the Longitudinal Health Insurance Database (LHID), was used in this study. The 2010 LHID is a subset of the National Health Insurance Database, which is released by the National Health Research Institutes in Taiwan. The 2010 LHIRD, in which 1 million beneficiaries are randomly sampled from National Health Insurance (NHI) system, comprises the claims data of outpatient, admission, and prescription from 2009 to 2013. The NHI system is a single-payer social insurance system, and the coverage was approximately $98 \%$ in 2010. To protect the privacy of patients and care providers, the personnel identification numbers are scrambled for de-identification. The study was approved by the Ethical Review Board of Chung Shan Medical University Hospital (CSMU No.: 18096).

\subsection{Patient Selection (Exposure of UTI Infection)}

This was a retrospective cohort study. We identified patients $(n=140,308)$ who visited a hospital or were hospitalized for UTI (ICD-9: 599.0, 595.0, 595.9, and 590) and had antibiotic therapy (cephalosporins, quinolone antibacterial, sulfonamides and trimethoprim, ampicillin, amoxicillin, and nitrofurantoin) in the same visit during 2009-2013 as the exposure group. To ensure that only newly identified UTI was included, prevalent cases of UTI in 2009 were excluded. Furthermore, patients newly diagnosed with UTI in 2013 were excluded because they were only followed up for less than 1 year. The index date was the first date of UTI visit or admission; additional exclusion criteria included patients aged less than 20 years old, those having any cancer, those who died, and those who underwent urine examination within 6 months after the index date. A total of 38,084 patients were diagnosed with UTI in this study. 


\subsection{Propensity Score Matching (PSM)}

A potential confounding bias exists in the observational study design. Propensity score matching (PSM) was used to diminish this bias. We used a logistic regression model to estimate the probability (propensity score) of UTI, using such predictors as age, the Charlson Comorbidity Index (CCI), and other comorbidities (hypertension (ICD-9: 401-405), diabetes (ICD-9: 250), dyslipidemia (ICD-9: 272), rheumatic diseases (ICD-9: 714, 710, 720, 696.0, and 696.1), coronary artery disease (ICD-9: 410-414), chronic obstructive pulmonary disease (COPD, ICD-9: 490-492, 493-496), and chronic kidney disease (CKD, ICD-9: 585)). The control (non-UTI exposure during 2009-2013) and study groups were 1:2 propensity-score-matched on the basis of sex because of the specific cancer sites in different genders. The greedy algorithm of PSM was applied using SAS macro [11].

\subsection{Outcome Measurement of Cancer Event}

Subsequent cancer events were identified according to ICD-9: 140-208 for $\geq 2$ outpatient visits or $\geq 1$ admission. Major cancer sites reported in Taiwan were considered for subevent analysis, including colorectal (ICD-9: 153-154), liver (ICD-9: 155), lung (ICD-9: 162), and breast (ICD-9: 174), bladder (ICD-9: 188), kidney (ICD-9: 189), male genital organs (ICD-9: 185-187), female genital organs (ICD-9: 179-184), and prostate (ICD-9: 185) cancers. All individuals were followed up from the index date until diagnosed with any cancer, death, or end of study (31 December 2013).

\subsection{Statistical Analysis}

All analyses were performed after stratifying the data by sex because gender-specific cancer sites were analyzed in this study. Chi-squared test was used to analyze the homogeneity of category variables, and univariate and multivariate Cox regression models were conducted to estimate the crude and adjusted hazard ratio (aHR) (95\% confidence interval (CI)). All statistical analyses were performed using SAS (version 9.4; SAS Institute, Cary, NC, USA). $p$ less than 0.05 indicated statistical significance.

\section{Results}

We identified 38,084 patients diagnosed with UTI from 2010 to 2012 and a total of 76,168 propensity score-matched controls to explore their sequential cancer risk after the index date (Figure 1). The baseline characteristics among the UTI and non-UTI groups stratified by sex are listed in Table 1 . After PSM, no significant difference was observed in the distributions of age group, CCI group, and comorbidities (hypertension, diabetes hyperlipidemia, rheumatic diseases, coronary artery disease, chronic obstructive pulmonary disease (COPD), and chronic kidney disease (CKD) in both genders. More female UTI cases (the sex ratio, F:M $=31,172: 6,912$ ) were observed, especially in women aged 20-44 and 45-65 years. The median of follow-up time was 25 months (Max: 42), because the index date (start point of follow-up) was 6 months after UTI.

The age-sex stratified incidence rate (per 10,000 person-months) and adjusted hazard risks of specific-site cancer (colorectal, liver, lung, genital organs, bladder, kidney, male prostate, female breast cancer, and any cancer) in patients with UTI are presented in Table 2. For men aged 20-64 years, significant aHRs were observed in any cancers ( $\mathrm{aHR}=1.37,95 \% \mathrm{CI}=1.02-1.86)$, bladder cancer $(\mathrm{aHR}=12.10,95 \% \mathrm{CI}=2.70-54.19)$, and kidney cancer $(\mathrm{aHR}=5.20,95 \% \mathrm{CI}=1.01-26.82)$. For men aged $\geq 65$ years, the associations were observed in any cancer $(\mathrm{aHR}=1.29,95 \% \mathrm{CI}=1.08-1.54)$, colorectal cancer $(\mathrm{aHR}=1.59,95 \% \mathrm{CI}=1.01-2.52)$, genital organ cancer $(\mathrm{aHR}=2.37,95 \% \mathrm{CI}=1.55-3.64)$, bladder cancer $(\mathrm{aHR}=28.60,95 \% \mathrm{CI}=6.80-120.28)$, kidney cancer $(\mathrm{aHR}=3.85,95 \% \mathrm{CI}=1.42-10.42)$, and prostate cancer $(\mathrm{aHR}=2.44,95 \% \mathrm{CI}=1.59-3.74)$. For women aged 20-64 years, the significant aHRs were estimated in liver cancer $(\mathrm{aHR}=2.44,95 \% \mathrm{CI}=1.59-3.74)$, bladder cancer $(\mathrm{aHR}=30.02$, $95 \% \mathrm{CI}=3.97-227.28)$, and kidney cancer $(\mathrm{aHR}=2.90,95 \% \mathrm{CI}=1.24-6.78)$. For women aged $\geq 65$ years, the significantly increased aHRs were observed in any cancer $(\mathrm{aHR}=1.30,95 \% \mathrm{CI}=1.11-1.53)$, liver 
cancer $(\mathrm{aHR}=1.54,95 \% \mathrm{CI}=1.02-2.33)$, bladder cancer $(\mathrm{aHR}=2.33,95 \% \mathrm{CI}=1.01-5.42)$, and kidney cancer $(\mathrm{aHR}=3.40,95 \% \mathrm{CI}=1.34-8.64)$.

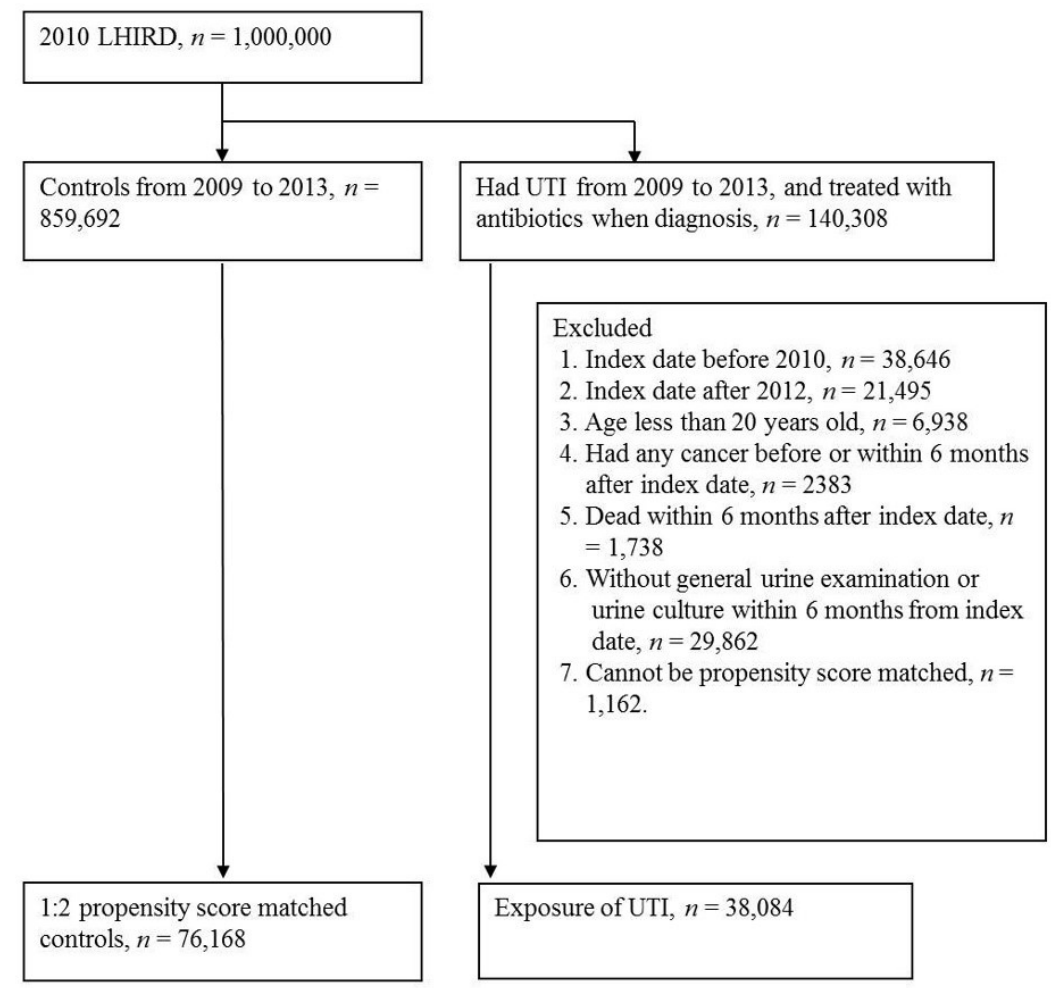

Figure 1. Flow chart for patient selection.

Table 1. Baseline characteristics of the study population.

\begin{tabular}{|c|c|c|c|c|c|c|}
\hline & \multicolumn{2}{|c|}{ Male } & \multirow[b]{2}{*}{$p$ Value } & \multicolumn{2}{|c|}{ Female } & \multirow[b]{2}{*}{$p$ Value } \\
\hline & $\begin{array}{c}\text { Non-UTI N = } \\
13,824\end{array}$ & UTI N = 6,912 & & $\begin{array}{c}\text { Non-UTI N = } \\
62,344\end{array}$ & $\begin{array}{c}\text { UTI N = } \\
31,172\end{array}$ & \\
\hline Age & & & 0.8332 & & & 0.4579 \\
\hline $20-44$ & $4355(31.5 \%)$ & $2201(31.84 \%)$ & & $31112(49.90 \%)$ & $15513(49.77 \%)$ & \\
\hline $45-65$ & 4411 (31.91\%) & 2209 (31.96\%) & & 20540 (32.95\%) & $10212(32.76 \%)$ & \\
\hline$\geq 65$ & $5058(36.59 \%)$ & $2502(36.20 \%)$ & & $10692(17.15 \%)$ & $5447(17.47 \%)$ & \\
\hline CCIs & & & 0.8214 & & & 0.1808 \\
\hline 0 & $6503(47.04 \%)$ & $3260(47.16 \%)$ & & $37784(60.61 \%)$ & $18906(60.65 \%)$ & \\
\hline $1-2$ & $5215(37.72 \%)$ & $2604(37.67 \%)$ & & $20116(32.27 \%)$ & $9961(31.95 \%)$ & \\
\hline $3-4$ & $1670(12.08 \%)$ & $846(12.24 \%)$ & & $3765(6.04 \%)$ & $1921(6.16 \%)$ & \\
\hline$\geq 5$ & $436(3.15 \%)$ & $202(2.92 \%)$ & & $679(1.09 \%)$ & $384(1.23 \%)$ & \\
\hline \multicolumn{7}{|l|}{ Co-morbidity } \\
\hline Hypertension & $5270(38.12 \%)$ & $2661(38.50 \%)$ & 0.5993 & 13847 (22.21\%) & $6963(22.34 \%)$ & 0.6605 \\
\hline Diabetes & $2484(17.97 \%)$ & $1248(18.06 \%)$ & 0.8781 & $7391(11.86 \%)$ & 3730 (11.97\%) & 0.6221 \\
\hline Hyperlipidemia & $2842(20.56 \%)$ & $1414(20.46 \%)$ & 0.8648 & $10363(16.62 \%)$ & $5189(16.65 \%)$ & 0.9258 \\
\hline Rheumatic diseases & $399(2.89 \%)$ & 207 (2.99\%) & 0.6619 & $2650(4.25 \%)$ & $1317(4.22 \%)$ & 0.8544 \\
\hline Coronary artery disease & $1943(14.06 \%)$ & $957(13.85 \%)$ & 0.6814 & $4702(7.54 \%)$ & $2367(7.59 \%)$ & 0.7795 \\
\hline COPD & $2083(15.07 \%)$ & $1047(15.15 \%)$ & 0.8801 & $4970(7.97 \%)$ & $2594(8.32 \%)$ & 0.0645 \\
\hline CKD & $470(3.40 \%)$ & $231(3.34 \%)$ & 0.8279 & $723(1.16 \%)$ & $397(1.27 \%)$ & 0.1312 \\
\hline Pneumonia & $254(1.84 \%)$ & $628(9.09 \%)$ & $<0.0001$ & $388(0.62 \%)$ & $716(2.30 \%)$ & $<0.0001$ \\
\hline
\end{tabular}

UTI: urinary tract infection; CCI: Charlson Comorbidity Index. 
Table 2. Age stratified cancer incidence rate (per 10,000 person months) and adjusted hazard ratio $(95 \% \text { C.I. })^{\dagger}$ in patients with UTI exposure by cancer site.

\begin{tabular}{|c|c|c|c|c|c|c|}
\hline & \multirow{2}{*}{\multicolumn{2}{|c|}{$\begin{array}{c}20-64 \text { y/o } \\
\text { Incidence Rate }\end{array}$}} & \multirow{3}{*}{ aHR } & \multicolumn{2}{|c|}{$\geq 65$ y/o } & \multirow{3}{*}{ aHR } \\
\hline & & & & Incidenc & Rate & \\
\hline & Non-UTI & UTI & & Non-UTI & UTI & \\
\hline \multicolumn{7}{|l|}{ Male } \\
\hline All cancer & 5.05 & 6.93 & $1.37(1.02-1.86)$ & 27.70 & 35.40 & $1.29(1.08-1.54)$ \\
\hline Colorectal & 0.58 & 0.77 & $1.32(0.54-3.23)$ & 3.78 & 5.96 & $1.59(1.01-2.52)$ \\
\hline Liver & 0.72 & 0.77 & $1.05(0.44-2.47)$ & 4.04 & 2.61 & $0.65(0.36-1.19)$ \\
\hline Lung & 0.67 & 0.77 & $1.46(0.75-2.86)$ & 5.98 & 4.10 & $1.13(0.75-1.70)$ \\
\hline Genital organs & 0.48 & 0.48 & 1.05 (0.36-3.09) & 3.52 & 8.39 & $2.37(1.55-3.64)$ \\
\hline Bladder & 0.10 & 1.15 & $12.10(2.70-54.19)$ & 0.18 & 5.03 & $28.60(6.80-120.28)$ \\
\hline Kidney & 0.10 & 0.48 & $5.20(1.01-26.82)$ & 0.53 & 2.05 & $3.85(1.42-10.42)$ \\
\hline Prostate & 0.43 & 0.38 & $0.96(0.29-3.15)$ & 3.43 & 8.39 & $2.44(1.59-3.74)$ \\
\hline \multicolumn{7}{|l|}{ Female } \\
\hline All cancer & 3.52 & 3.95 & $1.13(0.97-1.32)$ & 14.49 & 18.96 & $1.30(1.11-1.53)$ \\
\hline Colorectal & 0.45 & 0.32 & $0.70(0.42-1.17)$ & 2.76 & 2.91 & $1.06(0.71-1.57)$ \\
\hline Liver & 0.21 & 0.36 & $1.75(1.00-3.04)$ & 1.94 & 3.06 & $1.54(1.02-2.33)$ \\
\hline Lung & 0.25 & 0.47 & $1.22(0.86-1.71)$ & 2.56 & 2.52 & $0.75(0.43-1.32)$ \\
\hline Genital organs & 0.46 & 0.36 & $0.79(0.49-1.28)$ & 0.74 & 1.38 & $1.86(0.98-3.56)$ \\
\hline Bladder & 0.01 & 0.24 & $30.02(3.97-227.28)$ & 0.39 & 0.92 & $2.34(1.01-5.42)$ \\
\hline Kidney & 0.07 & 0.21 & $2.90(1.24-6.78)$ & 0.27 & 0.92 & $3.40(1.34-8.64)$ \\
\hline Breast & 1.16 & 1.04 & $0.90(0.68-1.21)$ & 1.51 & 1.68 & $1.10(0.65-1.85)$ \\
\hline
\end{tabular}

${ }^{\dagger}$ adjusted for age (per 1 year), CCI score, and co-morbidities (including hypertension, diabetes, hyperlipidemia, rheumatic disease, coronary artery disease, COPD, CKD, and pneumonia. Bold font indicates statistical significance $(p<0.05)$.

The significantly increased HRs of any cancer for UTI exposure in men (crude HR $=1.27$, $95 \% \mathrm{CI}=1.09-1.48 ; \mathrm{aHR}=1.32,95 \% \mathrm{CI}=1.12-1.54$ ) and women (crude $\mathrm{HR}=1.21,95 \% \mathrm{CI}=1.09-1.36$; $\mathrm{aHR}=1.21,95 \% \mathrm{CI}=1.08-1.35$ ) are indicated in Tables 3 and 4 . Additionally, we also demonstrated that in individuals aged $\geq 65$ years, a CCI score of $\geq 5$ indicated higher risk of cancer incidence.

Table 3. Adjusted hazard ratio of all cancer in males.

\begin{tabular}{ccccccc}
\hline & Crude HR & $\mathbf{9 5 \%}$ C.I. & $p$ Value & aHR $^{+}$ & 95\% C.I. & $p$ Value \\
\hline $\begin{array}{c}\text { UTI (Ref: No) } \\
\text { Yes }\end{array}$ & 1.27 & $1.09-1.48$ & 0.0023 & 1.32 & $1.12-1.54$ & 0.0007 \\
\hline Age (Ref: 20-44) & & & & & & \\
\hline $45-65$ & 5.21 & $3.50-7.76$ & $<0.0001$ & 4.69 & $3.14-7.03$ & $<0.0001$ \\
$\geq 65$ & 16.37 & $11.26-23.79$ & $<0.0001$ & 12.32 & $8.29-18.31$ & $<0.0001$ \\
\hline CCIs score (Ref: 0) & & & & & & \\
\hline $1-2$ & 2.34 & $1.94-2.82$ & $<0.0001$ & 1.28 & $1.03-1.58$ & 0.0230 \\
$3-4$ & 3.87 & $3.11-4.81$ & $<0.0001$ & 1.50 & $1.13-1.99$ & 0.0046 \\
$\geq 5$ & 5.42 & $3.92-7.49$ & $<0.0001$ & 1.88 & $1.24-2.84$ & 0.0027 \\
\hline Co-morbidity & & & & & & \\
\hline Hypertension & 2.69 & $2.31-3.14$ & $<0.0001$ & 1.12 & $0.94-1.34$ & 0.1901 \\
Diabetes & 1.76 & $1.49-2.09$ & $<0.0001$ & 0.90 & $0.73-1.10$ & 0.2867 \\
Hyperlipidemia & 1.38 & $1.16-1.64$ & 0.0002 & 0.93 & $0.77-1.12$ & 0.4413 \\
Rheumatic diseases & 1.31 & $0.89-1.94$ & 0.1747 & 1.00 & $0.67-1.48$ & 0.9981 \\
Coronary artery disease & 2.44 & $2.06-2.89$ & $<0.0001$ & 1.25 & $1.04-1.49$ & 0.0165 \\
COPD & 2.02 & $1.70-2.40$ & $<0.0001$ & 1.05 & $0.86-1.27$ & 0.6569 \\
CKD & 2.21 & $1.62-3.03$ & $<0.0001$ & 0.98 & $0.70-1.37$ & 0.8845 \\
Pneumonia & 2.15 & $1.61-2.88$ & $<0.0001$ & 0.95 & $0.70-1.29$ & 0.7612 \\
\hline
\end{tabular}

+ adjusted for UTI infection, age group, CCI score, and co-morbidities (including hypertension, diabetes, hyperlipidemia, rheumatic disease, coronary artery disease, COPD, CKD, and pneumonia. 
Table 4. Adjusted hazard ratio of all cancer in Female.

\begin{tabular}{ccccccc}
\hline & Crude HR & $\mathbf{9 5 \%}$ C.I. & $p$ Value & aHR $^{+}$ & 95\% C.I. & $p$ Value \\
\hline $\begin{array}{c}\text { UTI (Ref: No) } \\
\text { Yes }\end{array}$ & 1.21 & $1.09-1.36$ & 0.0007 & 1.21 & $1.08-1.35$ & 0.0009 \\
\hline Age (Ref: 20-44) & & & & & & \\
\hline $45-65$ & 4.68 & $3.94-5.57$ & $<0.0001$ & 4.41 & $3.68-5.27$ & $<0.0001$ \\
$\geq 65$ & 10.78 & $9.09-12.78$ & $<0.0001$ & 9.00 & $7.37-11.00$ & $<0.0001$ \\
\hline CCIs score (Ref: 0) & & & & & & \\
\hline $1-2$ & 2.16 & $1.92-2.44$ & $<0.0001$ & 1.24 & $1.08-1.43$ & 0.0023 \\
$3-4$ & 3.86 & $3.27-4.57$ & $<0.0001$ & 1.40 & $1.13-1.74$ & 0.0024 \\
$\geq 5$ & 5.47 & $4.02-7.44$ & $<0.0001$ & 1.54 & $1.03-2.29$ & 0.0349 \\
\hline Co-morbidity & & & & & & \\
\hline Hypertension & 3.08 & $2.76-3.43$ & $<0.0001$ & 1.11 & $0.97-1.26$ & 0.1444 \\
Diabetes & 2.39 & $2.10-2.71$ & $<0.0001$ & 0.95 & $0.81-1.11$ & 0.4982 \\
Hyperlipidemia & 1.94 & $1.72-2.19$ & $<0.0001$ & 0.88 & $0.78-1.01$ & 0.0774 \\
Rheumatic diseases & 1.28 & $1.00-1.63$ & 0.0478 & 0.93 & $0.72-1.18$ & 0.5332 \\
Coronary artery disease & 2.51 & $2.18-2.91$ & $<0.0001$ & 1.05 & $0.90-1.23$ & 0.5270 \\
COPD & 1.63 & $1.39-1.93$ & $<0.0001$ & 0.91 & $0.76-1.09$ & 0.3035 \\
CKD & 3.76 & $2.84-4.97$ & $<0.0001$ & 1.51 & $1.09-2.10$ & 0.0141 \\
Pneumonia & 2.18 & $1.52-3.13$ & $<0.0001$ & 0.97 & $0.67-1.41$ & 0.8892 \\
\hline
\end{tabular}

+ adjusted for UTI infection, age group, CCI score, and co-morbidities (including hypertension, diabetes, hyperlipidemia, rheumatic disease, coronary artery disease, COPD, CKD, and pneumonia.

In Table 5, no interaction effect between UTI and pneumonia on cancer incidence was observed. We demonstrated the dose response of antibiotic prescriptions on cancer incidence in men; the significant $\mathrm{p}$ for trend was observed in any cancer $(p=0.0130)$ and bladder cancer $(p=0.0066)$. However, no significant $p$ trends were observed in women (Table 6).

The Kaplan-Meier curve for specific cancer risk among the study groups are shown in Appendix A Figure A1. Any cancer risk was under proportional hazard assumption; however, bladder and kidney cancer risks were modified after 24 and 18 months, respectively, in the elder ( $\geq 65$ years old) population.

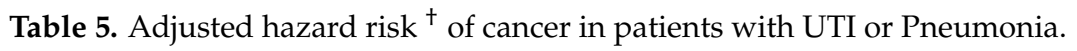

\begin{tabular}{|c|c|c|c|c|c|}
\hline & $\begin{array}{l}\text { Non-UTI and } \\
\text { Non-Pneumonia }\end{array}$ & Only UTI & $\begin{array}{c}\text { Only } \\
\text { Pneumonia }\end{array}$ & $\begin{array}{l}\text { UTI Combined } \\
\text { Pneumonia }\end{array}$ & $\begin{array}{c}p \text { for } \\
\text { Interaction }\end{array}$ \\
\hline \multicolumn{6}{|l|}{ Male } \\
\hline All cancer & Reference & 1.35 (1.15-1.59) & $1.24(0.75-2.06)$ & $1.14(0.80-1.64)$ & 0.2270 \\
\hline Colorectal & Reference & $1.54(0.99-2.38)$ & $1.90(0.58-6.19)$ & $1.76(0.75-4.16)$ & 0.4963 \\
\hline Liver & Reference & $0.86(0.52-1.41)$ & $1.12(0.27-4.65)$ & $0.23(0.03-1.69)$ & 0.2585 \\
\hline Lung & Reference & $0.79(0.50-1.24)$ & $1.32(0.48-3.68)$ & $0.78(0.31-1.93)$ & 0.6723 \\
\hline Genital organs & Reference & $2.23(1.49-3.35)$ & $0.60(0.08-4.36)$ & $1.25(0.49-3.17)$ & 0.9541 \\
\hline Bladder & Reference & $21.64(7.71-60.77)$ & Can not estimate & $7.63(1.37-42.42)$ & 0.9879 \\
\hline Kidney & Reference & $4.10(1.72-9.80)$ & Can not estimate & $3.84(0.78-18.87)$ & 0.9908 \\
\hline Prostate & Reference & $2.29(1.51-3.45)$ & $0.61(0.08-4.45)$ & $1.28(0.51-3.26)$ & 0.9426 \\
\hline \multicolumn{6}{|l|}{ Female } \\
\hline All cancer & Reference & $1.20(1.08-1.35)$ & $0.85(0.42-1.72)$ & $1.24(0.81-1.90)$ & 0.6554 \\
\hline Colorectal & Reference & $0.92(0.68-1.26)$ & $0.68(0.10-4.93)$ & $0.35(0.05-2.50)$ & 0.6741 \\
\hline Liver & Reference & $1.57(1.12-2.21)$ & $0.72(0.10-5.19)$ & $1.76(0.70-4.41)$ & 0.6862 \\
\hline Lung & Reference & $1.26(0.91-1.75)$ & $2.13(0.676 .79)$ & $1.95(0.78-4.85)$ & 0.6684 \\
\hline Genital organs & Reference & $1.03(0.70-1.52)$ & Can not estimate & 1.63 (0.39-6.77) & 0.9735 \\
\hline Bladder & Reference & $4.84(2.39-9.80)$ & Can not estimate & $4.60(0.58-36.22)$ & 0.9944 \\
\hline Kidney & Reference & $3.43(1.81-6.51)$ & $6.44(0.83-50.19)$ & Can not estimate & 0.9768 \\
\hline Breast & Reference & $0.96(0.75-1.24)$ & $0.79(0.11-5.66)$ & $0.43(0.06-3.08)$ & 0.6890 \\
\hline
\end{tabular}

${ }^{\dagger}$ adjusted for age group, CCI score, and co-morbidities (including hypertension, diabetes, hyperlipidemia, rheumatic disease, coronary artery disease, COPD, CKD, and pneumonia 
Table 6. Adjusted hazard risk ${ }^{\dagger}$ of cancer for dosage (days) trend of antibiotics prescriptions in patients with UTI.

\begin{tabular}{|c|c|c|c|c|}
\hline & \multicolumn{3}{|c|}{ Days of Antibiotics Prescriptions within 6 Months before Index Date } & \multirow{2}{*}{$p$ for Trend } \\
\hline & 1-6 days & 7-13 days & $\geq 14$ days & \\
\hline Male & $n=17241$ & $n=1940$ & $n=1555$ & \\
\hline All cancer & Reference & $1.02(0.75-1.40)$ & $1.45(1.09-1.91)$ & 0.0130 \\
\hline Colorectal & Reference & $1.16(0.54-2.46)$ & $1.05(0.49-2.23)$ & 0.8669 \\
\hline Liver & Reference & $0.93(0.32-2.72)$ & $1.35(0.51-3.56)$ & 0.5874 \\
\hline Lung & Reference & $1.43(0.60-3.41)$ & $1.39(0.58-3.30)$ & 0.4213 \\
\hline Genital organs & Reference & $0.81(0.37-1.77)$ & $1.44(0.78-2.66)$ & 0.2860 \\
\hline Bladder & Reference & $1.23(0.50-3.02)$ & $2.73(1.32-5.64)$ & 0.0066 \\
\hline Kidney & Reference & $0.68(0.18-2.55)$ & $1.14(0.37-3.54)$ & 0.8995 \\
\hline Prostate & Reference & $0.82(0.38-1.78)$ & $1.36(0.73-2.54)$ & 0.3822 \\
\hline Female & $n=80515$ & $n=9014$ & $n=3987$ & \\
\hline All cancer & Reference & $1.08(0.88-1.32)$ & $1.10(0.87-1.40)$ & 0.3605 \\
\hline Colorectal & Reference & $1.00(0.54-1.85)$ & $1.31(0.68-2.54)$ & 0.4775 \\
\hline Liver & Reference & $1.19(0.67-2.10)$ & $1.12(0.58-2.19)$ & 0.6352 \\
\hline Lung & Reference & $1.53(0.87-2.69)$ & $1.49(0.78-2.85)$ & 0.1511 \\
\hline Genital organs & Reference & $2.06(1.10-3.85)$ & $0.33(0.08-1.41)$ & 0.7254 \\
\hline Bladder & Reference & $0.41(0.12-1.44)$ & $2.30(1.01-5.25)$ & 0.1283 \\
\hline Kidney & Reference & $2.34(0.97-5.66)$ & $1.73(0.58-5.20)$ & 0.1741 \\
\hline Breast & Reference & $1.13(0.70-1.83)$ & $1.42(0.81-2.49)$ & 0.2356 \\
\hline
\end{tabular}

${ }^{+}$adjusted for age group, $\mathrm{CCI}$ score, and co-morbidities (including hypertension, diabetes, hyperlipidemia, rheumatic disease, coronary artery disease, $\mathrm{COPD}, \mathrm{CKD}$, and pneumonia.

\section{Discussion}

Per the analysis results presented in Figure A1, risk significantly increased not only in GUC but also in any-cancer development. Males older than 65 years exhibited a negative association between UTI and lung cancer risk (Figure A1). In previous reports, cephalosporins and quinolones were associated with antitumor properties [12,13]. The incidence of lung cancer in middle-aged men with UTI may be reduced through antibiotic treatments, and this hypothesis requires more evidence before it can be confirmed.

UTI and pneumonia are clinically common complications, and the results of this work also showed a high correlation between UTI and pneumonia $(p<0.001)$ (Table 3). Some reports have revealed that pneumonia has a high correlation with lung cancer, and Marcus et al. provided evidence of increased lung cancer risk among history of pneumonia rather than immunodeficiency [14]. The association between pneumonia and any cancer is rarely reported. The incidence of association between pneumonia and any cancer was low in both men and women in this study $(p>0.05)$.

UTI is particularly associated with the bladder and kidney cancer in both men and women (Table 2). The significant and large relative risk was found whether in young (20-64 y/o) or elderly ( $\geq 65 \mathrm{y} / \mathrm{o}$ ) population. This evidence reinforces the study hypothesis that UTI directly increase the risk in bladder and kidney cancers, which is consistent with Anderson-Otunu's 2016 report [6]. Vermeulen (2015) revealed that UTI are associated with a high risk of urinary bladder cancer in postmenopausal women, especially in women who smoke or had smoked [7]. In males, the obstructive urinary symptoms induced by benign prostatic hyperplasia include difficulty in urination and urine retention, resulting in UTI caused from urinary stasis [15].

Similarly, Table 6 indicates that the use of antibiotics in the course of UTI treatments increases the risk of bladder and kidney cancers. We analyzed the tumor incidence risk between UTI and pneumonia after antibiotic treatment (Table 5). The correlation coefficient between antibiotic treatment and tumor incidence was considerably low. However, a significant increase was observed in the incidence of bladder cancer in men after antibiotic treatment for more than 7 days (Table 6). These results are contrary to many research conclusions, and most antibiotics 
such as cephalosporins, quinolones, and ampicillins are considered as a kind of cancer treatment drug rather than a carcinogen $[12,13,16]$. However, no report has clarified whether beta-lactams, amoxicillin, sulfamethoxazole, and trimethoprim are carcinogenic or have antitumor properties. Only nitrofurantoin is a possible carcinogen due to its genotoxic and carcinogenic potential structures, despite its antimicrobial property [17-20]. In Kimura's report (2016), nitrofurantoin does not exert sufficient renal carcinogen responses even after 28 days of administration [21]. In a recent study on the structure-related genotoxicity of nitrofurantoin, a new evidence revealed that nitrofurantoin does not increase the mutation frequency in the experimental mice. Nevertheless, nitrofurantoin presents no genotoxicity without oxidative stress [22]. This report provided a safe basis for nitrofuran compound development. The gold standard for UTI diagnosis is isolation and quantification of pathogens in the presence of symptoms and obtaining the antibiotic sensitivity pattern to allow specific treatments. However, in this observational study, we cannot provide the evidence of carcinogen from antibiotics. The length (days) of antibiotics usage also correlated with the severity of infection, that might be the risk factor of cancer. However, the appropriate drug dosage should be based on the severity, characteristics and treatment of the infection situation. Therefore, we should consider antibiotic doses in the treatment of UTI under the premise of controlling infection and avoiding drug resistance. Thus, further research needs to clarify this issue in the future. Women after UTI have higher liver cancer risk as reported in this work, especially approximately 30 months after infection. UTI infection is positively associated with GUC, which is consistent with previous findings. Therefore, this study posits a nonpathogenic causal relationship with UTI symptoms.

Our research has some limitations. First, the database used does not contain information on patients' clinical presentation, for example, the severity of UTI, personal behavioral information such as drinking, smoking habits, and body mass index, and microbiological culture data that might affect UTI occurrence. Second, the NHI system is limited to the population of Taiwan. Our findings reflect the situation in Taiwan, but it may not be applicable to Western populations. These confounding factors might have influenced the results.

\section{Conclusions}

In conclusion, UTI are highly correlated with the incidence of all tumors. Clinicians should therefore refer to tumors screening in UTI patients, especially older patients, and not just patients with GUC. In UTI treatment, especially when multiple pathogenic factors are entailed, antibiotics must be used cautiously, and the time and dose of antibiotics should be minimized. Because UTI may increase the incidence of genital organ, bladder, kidney, male colorectal, prostate, and female liver cancer, knowledge about preventing UTI such as proper drinking water, exercise, and toilet habits should be enhanced in educating the general public.

Author Contributions: Conceptualization, C.-H.H., Y.-H.C., S.-F.Y. and C.-B.Y.; Validation, Y.-H.C. and C.-B.Y.; Formal Analysis, H.-W.Y. and J.-Y.H.; Writing-Original Draft Preparation, C.-H.H., S.-F.Y. and C.-B.Y.; Writing-Review and Editing, C.-H.H., S.-F.Y. and C.-B.Y.

Funding: This research received no external funding.

Acknowledgments: This study was partly based on data from the NHIRD provided by the NHI Administration, Ministry of Health and Welfare, and managed by the National Health Research Institutes (registration number: NHIRD-104-148). The interpretation and conclusions contained herein do not represent those of the NHI Administration, Ministry of Health and Welfare, or National Health Research Institutes.

Conflicts of Interest: The authors declare no conflict of interest. 


\section{Appendix A}
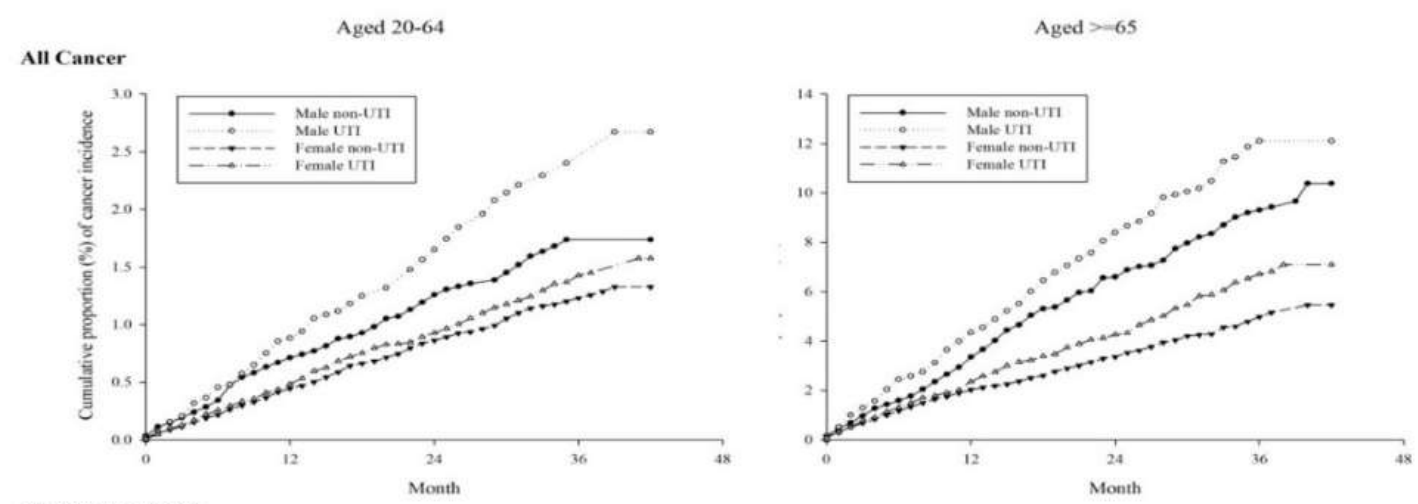

Colorectal Cancer
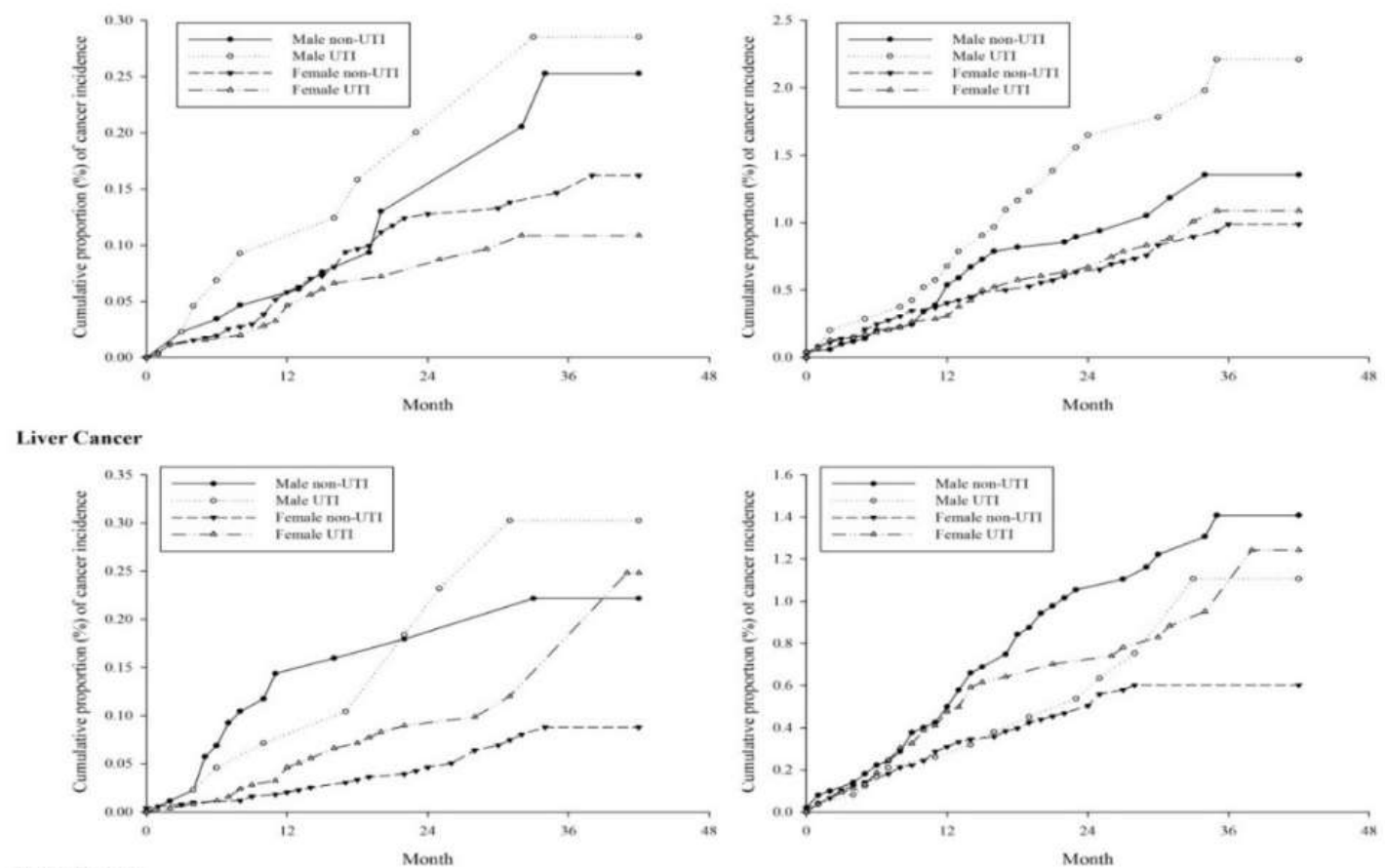

Lung Cancer
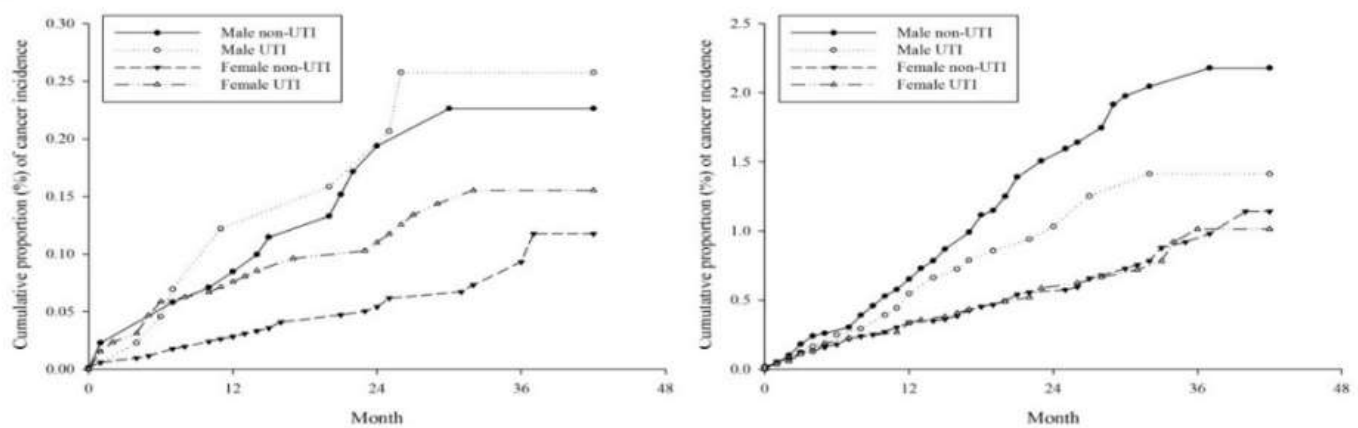

Figure A1. Cont. 

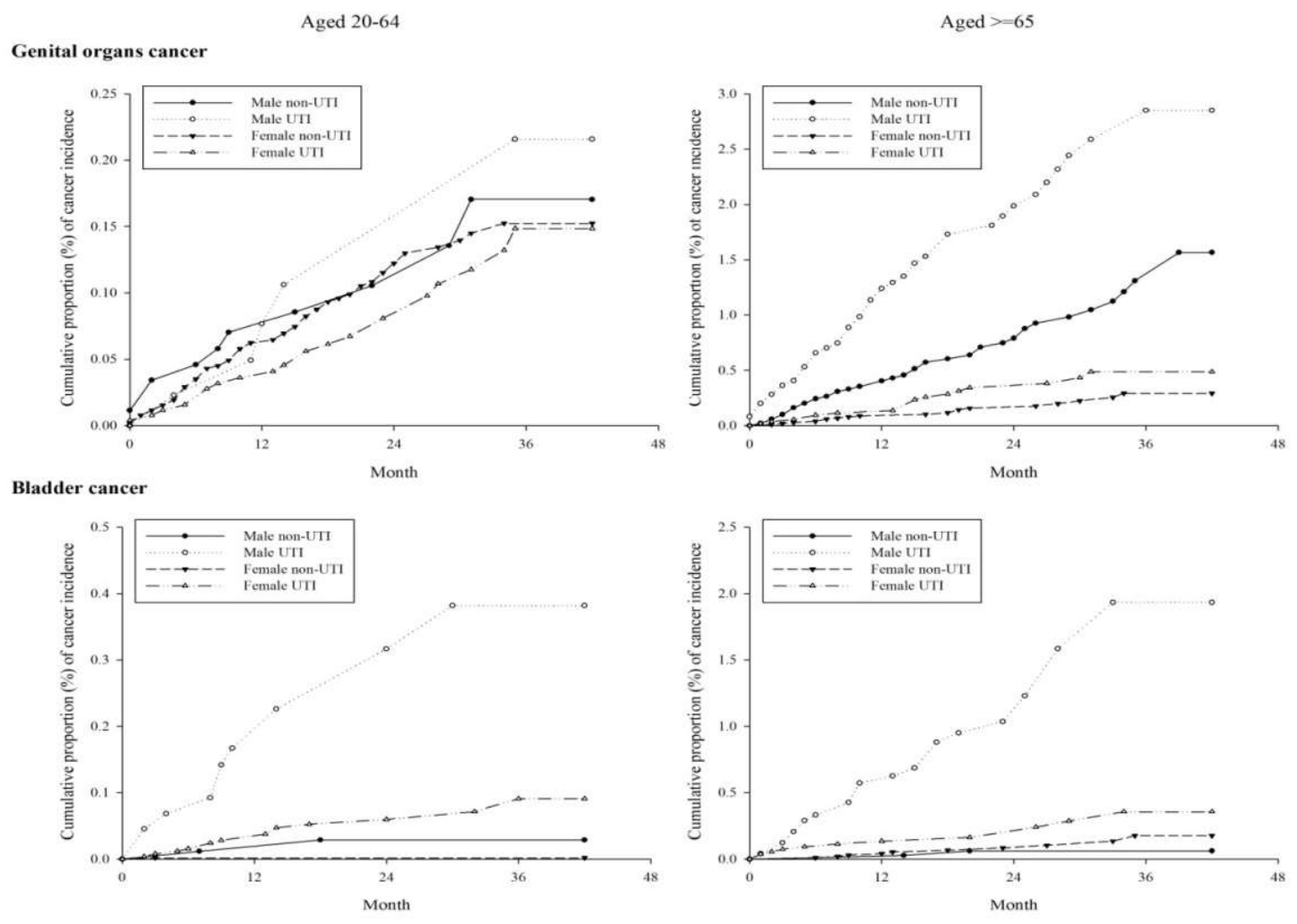

Kidney cancer
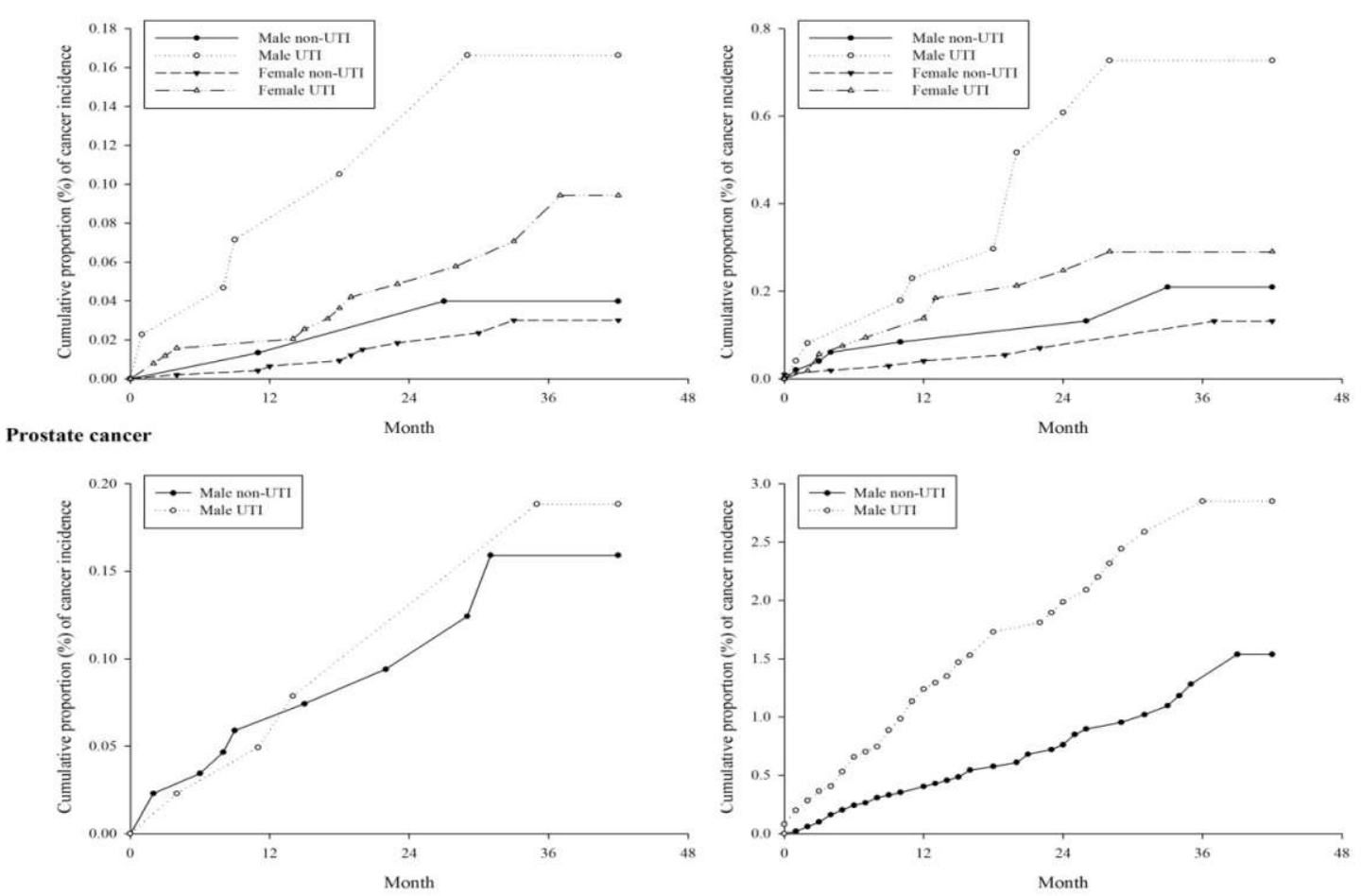

Figure A1. Cont. 

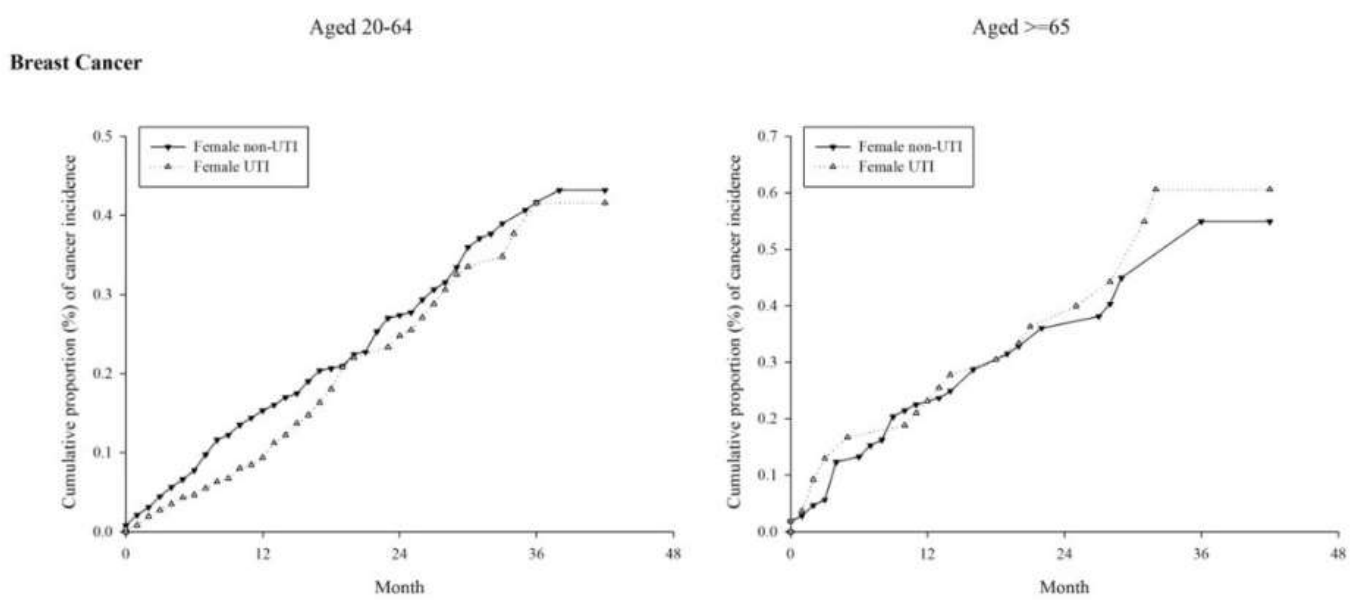

Figure A1. Kaplan-Meier curve for specific cancer risk in patients with/without UTI infection stratified by age group.

\section{References}

1. Nicolle, L.E. Urinary tract infection. Crit. Care Clin. 2013, 29, 699-715. [CrossRef] [PubMed]

2. Sun, L.M.; Lin, C.L.; Liang, J.A.; Liu, S.H.; Sung, F.C.; Chang, Y.J.; Kao, C.H. Urinary tract infection increases subsequent urinary tract cancer risk: A population-based cohort study. Cancer Sci. 2013, 104, 619-623. [CrossRef] [PubMed]

3. Erdem, I.; Kara Ali, R.; Ardic, E.; Elbasan Omar, S.; Mutlu, R.; Topkaya, A.E. Community-acquired lower urinary tract infections: Etiology, antimicrobial resistance, and treatment results in female patients. J. Glob. Infect. Dis. 2018, 10, 129-132. [CrossRef] [PubMed]

4. Arnold, J.J.; Hehn, L.E.; Klein, D.A. Common questions about recurrent urinary tract infections in women. Am. Family Phys. 2016, 93, 560-569.

5. Rowe, T.A.; Juthani-Mehta, M. Diagnosis and management of urinary tract infection in older adults. Infect. Disease Clin. N. Am. 2014, 28, 75-89. [CrossRef] [PubMed]

6. Anderson-Otunu, O.; Akhtar, S. Chronic infections of the urinary tract and bladder cancer risk: A systematic review. Asian Pac. J. Cancer Prev. APJCP 2016, 17, 3805-3807. [PubMed]

7. Vermeulen, S.H.; Hanum, N.; Grotenhuis, A.J.; Castano-Vinyals, G.; van der Heijden, A.G.; Aben, K.K.; Mysorekar, I.U.; Kiemeney, L.A. Recurrent urinary tract infection and risk of bladder cancer in the nijmegen bladder cancer study. Br. J. Cancer 2015, 112, 594-600. [CrossRef] [PubMed]

8. Dune, T.J.; Price, T.K.; Hilt, E.E.; Thomas-White, K.J.; Kliethermes, S.; Brincat, C.; Brubaker, L.; Schreckenberger, P.; Wolfe, A.J.; Mueller, E.R. Urinary symptoms and their associations with urinary tract infections in urogynecologic patients. Obstet. Gynecol. 2017, 130, 718-725. [CrossRef] [PubMed]

9. Russell, B.; Garmo, H.; Beckmann, K.; Stattin, P.; Adolfsson, J.; Van Hemelrijck, M. A case-control study of lower urinary-tract infections, associated antibiotics and the risk of developing prostate cancer using pcbase 3.0. PLoS ONE 2018, 13, e0195690. [CrossRef] [PubMed]

10. Chastain, D.B.; King, S.T.; Stover, K.R. Rethinking urinary antibiotic breakpoints: Analysis of urinary antibiotic concentrations to treat multidrug resistant organisms. BMC Res. Notes 2018, 11, 497. [CrossRef] [PubMed]

11. Parsons, L.S. Performing a 1: N case-control match on propensity score. In Proceedings of the 29th Annual SAS Users Group International Conference, Montreal, QC, Canada, 9-12 May 2004; p. 165-29.

12. Maj, M.; Bajek, A.; Nalejska, E.; Porowinska, D.; Kloskowski, T.; Gackowska, L.; Drewa, T. Influence of mesenchymal stem cells conditioned media on proliferation of urinary tract cancer cell lines and their sensitivity to ciprofloxacin. J. Cell. Biochem. 2017, 118, 1361-1368. [CrossRef] [PubMed]

13. Batalha, P.N.; Vieira de Souza, M.C.; Pena-Cabrera, E.; Cruz, D.C.; da Costa Santos Boechat, F. Quinolones in the search for new anticancer agents. Curr. Pharm. Des. 2016, 22, 6009-6020. [CrossRef] [PubMed]

14. Marcus, J.L.; Leyden, W.A.; Chao, C.R.; Horberg, M.A.; Klein, D.B.; Quesenberry, C.P., Jr.; Towner, W.J.; Silverberg, M.J. Immunodeficiency, aids-related pneumonia, and risk of lung cancer among hiv-infected individuals. AIDS 2017, 31, 989-993. [CrossRef] [PubMed] 
15. Michaud, D.S. Chronic inflammation and bladder cancer. Urol. Oncol. 2007, 25, 260-268. [CrossRef] [PubMed]

16. Ferraz, R.; Costa-Rodrigues, J.; Fernandes, M.H.; Santos, M.M.; Marrucho, I.M.; Rebelo, L.P.; Prudencio, C.; Noronha, J.P.; Petrovski, Z.; Branco, L.C. Antitumor activity of ionic liquids based on ampicillin. ChemMedChem 2015, 10, 1480-1483. [CrossRef] [PubMed]

17. Hasegawa, R.; Murasaki, G.; St John, M.K.; Zenser, T.V.; Cohen, S.M. Evaluation of nitrofurantoin on the two stages of urinary bladder carcinogenesis in the rat. Toxicology 1990, 62, 333-347. [CrossRef]

18. Kijima, A.; Ishii, Y.; Takasu, S.; Matsushita, K.; Kuroda, K.; Hibi, D.; Suzuki, Y.; Nohmi, T.; Umemura, T. Chemical structure-related mechanisms underlying in vivo genotoxicity induced by nitrofurantoin and its constituent moieties in gpt delta rats. Toxicology 2015, 331, 125-135. [CrossRef] [PubMed]

19. Williams, G.M. Classification of genotoxic and epigenetic hepatocarcinogens using liver culture assays. Ann. N. Y. Acad. Sci. 1980, 349, 273-282. [CrossRef]

20. International Agency for Research on Cancer. Monographs on the Evaluation of Carcinogen Risk to Humans: Some Industrial Chemicals; International Agency for Research on Cancer: Lyon, France, 1994.

21. Kimura, M.; Mizukami, S.; Watanabe, Y.; Hasegawa-Baba, Y.; Onda, N.; Yoshida, T.; Shibutani, M. Disruption of spindle checkpoint function in rats following 28 days of repeated administration of renal carcinogens. J. Toxicol. Sci. 2016, 41, 91-104. [CrossRef] [PubMed]

22. Tsuchiya, T.; Kijima, A.; Ishii, Y.; Takasu, S.; Yokoo, Y.; Nishikawa, A.; Yanai, T.; Umemura, T. Role of oxidative stress in the chemical structure-related genotoxicity of nitrofurantoin in nrf2-deficient gpt delta mice. J. Toxicol. Pathol. 2018, 31, 169-178. [CrossRef] [PubMed]

(C) 2019 by the authors. Licensee MDPI, Basel, Switzerland. This article is an open access article distributed under the terms and conditions of the Creative Commons Attribution (CC BY) license (http:/ / creativecommons.org/licenses/by/4.0/). 